\title{
Paul Grice und die Unterscheidung zwischen Semantik und Pragmatik
}

\section{1 \\ Einleitung}

Der Einfluss von Paul Grice wird häufig als der wichtigste unter den Faktoren identifiziert, die zum Rückgang der Beliebtheit der Ordinary LanguagePhilosophie beigetragen haben. Besonders deutlich wird diese Auffassung in Scott Soames' Philosophical Analysis in the Twentieth Century zum Ausdruck gebracht, in dem einer von sieben Teilen des zweiten Bandes mit »Paul Grice and the End of Ordinary Language Philosophy« überschrieben ist. ${ }^{1}$ Die Argumente von Grice, die viele Philosophinnen und Philosophen von der Inadäquatheit der Ordinary Language-Philosophie überzeugt haben, sind allerdings nicht gegen diese Philosophie-Konzeption selbst gerichtet, sondern gegen die ihr zugrunde liegende Konzeption sprachlicher Bedeutung: die so genannte »Gebrauchstheorie der Bedeutung«. Grices Einwand gegen diese Theorie besteht darin, dass sie bestimmte Phänomene zur Bedeutung von sprachlichen Ausdrücken rechnet, die eigentlich in den Bereich der Pragmatik gehören. Im vorliegenden Kapitel versuche ich, diesen Einwand zu entkräften.

Manche Philosophinnen und Philosophen sind sogar der Auffassung, die Gebrauchstheorie der Bedeutung lasse überhaupt keine Unterscheidung zwischen Semantik und Pragmatik zu. Woher diese Idee kommt, liegt auf der Hand: Die Semantik ist gemäß der Standard-Definition die Lehre der sprachlichen Bedeutung, die Pragmatik die Lehre des Sprachgebrauchs. Wenn die Vertreterinnen und Vertreter der Gebrauchstheorie die Bedeutung sprachlicher Ausdrücke mit deren Gebrauch identifizieren, gibt es für sie zwischen Semantik und Pragmatik also keinen Unterschied. Wie nachfolgend gezeigt werden soll, fällt der Gegenstandsbereich der Pragmatik allerdings nicht mit dem zusammen, was die Gebrauchstheorie mit der Bedeutung sprachlicher Ausdrücke gleichsetzt. Während sich die Gebrauchstheorie, grob gesagt, auf die etablierte oder korrekte Verwendung sprachlicher Ausdrücke bezieht, befasst sich die Pragmatik mit einzelnen Äußerungen sprachlicher Ausdrücke und mit allgemeinen, der Kommunikation zugrunde liegenden Prinzipien.

1 Vgl. auch Parker-Ryan (IEP). 
Die Beantwortung der Frage, ob die Gebrauchstheorie der Bedeutung dem Unterschied zwischen Semantik und Pragmatik Rechnung tragen kann bzw. ob die Gebrauchstheorie bestimmte pragmatische Phänomene der Semantik zuordnet, wird durch den Umstand verkompliziert, dass es eine Debatte darum gibt, wo die Grenze zwischen Semantik und Pragmatik zu ziehen: Es besteht keine Einigkeit darüber, welches Kriterium zur Unterscheidung von Semantik und Pragmatik angemessen ist. Es wäre also prinzipiell auch möglich, dass die Gebrauchstheorie eine Unterscheidung zulässt, die einem der verschiedenen Anwärter für die Unterscheidung zwischen Semantik und Pragmatik entspricht, dass sich jedoch herausstellt, dass die Grenze zwischen Semantik und Pragmatik idealerweise an einer anderen Stelle gezogen werden sollte und dass man auf der Grundlage der Gebrauchstheorie diese ideale Unterscheidung nicht nachvollziehen kann.

Das vorliegende Kapitel ist wie folgt aufgebaut: Im Abschnitt 1 wird auf die aktuelle Debatte zur Abgrenzung der Pragmatik von der Semantik eingegangen und darauf, wie in dieser Debatte auf die Gebrauchstheorie der Bedeutung Bezug genommen wird. Im Abschnitt 2 wird anhand eines Beispiels gezeigt, dass die Gebrauchstheorie pragmatische Phänomene jedenfalls nicht systematisch als semantisch fehl-klassifiziert. Im Abschnitt 3 wird untersucht, ob es gute Gründe dafür gibt, eine der in der entsprechenden Debatte vorgeschlagenen Weisen der Unterscheidung zwischen Semantik und Pragmatik den anderen Kandidaten vorzuziehen. Schließlich wird im Abschnitt 4 das Argument von Grice rekonstruiert und kritisiert, gemäß welchem die Gebrauchstheorie zu verwerfen ist, weil sie bestimmte Phänomene der Semantik zurechnet, die in Wirklichkeit pragmatischer Natur sind.

\section{Die gegenwärtige Debatte}

Zu den wichtigsten Publikationen, die in den letzten fünfzehn Jahren zur Abgrenzung zwischen Semantik und Pragmatik erschienen sind, gehören die Sammelbände The Semantics/Pragmatics Distinction (2004, hg. von C. Bianchi) und Semantics versus Pragmatics (2005, hg. von Z. G. Szabó) sowie die Monographien von Herman Cappelen und Ernie Lepore (Insensitive Semantics, 2004), von François Recanati (Truth-Conditional Pragmatics, 2010) und von Deirdre Wilson und Dan Sperber (Meaning and Relevance, 2012). Neben der allgemeinen Frage, wo die Grenze zwischen Semantik und Pragmatik adäquaterweise gezogen wird, werden in diesen Büchern sowie in zahlreichen Artikeln unter anderem die Probleme diskutiert, 
- wie man Grices Konzeptionen des Kooperationsprinzips und der Konversationsmaximen auffassen, erweitern oder verbessern muss, um alle Aspekte dessen zu erklären, was wir in Kommunikationssituationen (über die wörtliche Bedeutung des geäußerten Ausdrucks hinaus) zu vermitteln imstande sind, ${ }^{2}$

- in welchem Ausmaß der Kontext einer Äußerung deren Wahrheitsbedingungen beeinflusst, ${ }^{3}$

- inwiefern die semantische und die pragmatische Analyse einer Äußerung ineinandergreifen ${ }^{4}$ und

- wie sich die Kategorien des Gesagten und des Gemeinten (oder der »Sprecher-Bedeutung «) zur Semantik/Pragmatik-Unterscheidung verhalten. ${ }^{5}$ In diversen Texten nehmen die an der Debatte beteiligten Philosophinnen und Philosophen, Linguistinnen und Linguisten nebenbei auf die Gebrauchstheorie der Bedeutung oder auf die Ordinary Language-Philosophie Bezug und behaupten, den Vertreterinnen und Vertretern dieser Theorie resp. dieser Philosophie-Konzeption zufolge gebe es keinen Unterschied zwischen Semantik und Pragmatik. ${ }^{6}$ So schreiben zum Beispiel Zoltán Gendler Szabó und Kent Bach:

[T] hose who subscribe to the view that the meaning of a linguistic expression is identical to its use must reject the proposed distinction between semantics and pragmatics. ${ }^{7}$

Wittgenstein [...] came to think of language not as a system of representation but as a system of devices for engaging in various sorts of social activity; hence, "the meaning of a word is its use in the language" [...]. Here he went too far, for there is good reason to separate the theory of linguistic meaning (semantics) from the theory of language use (pragmatics) $[\ldots] .8$

2 Vgl. z. B. Turner 1997, Abschnitt 3.

3 Vgl. z. B. Cappelen/Lepore 2005.

4 Vgl. z. B. Levinson 2000, S. 236-243.

5 Vgl. z. B. Recanati 2001. Dabei handelt es sich natürlich nur dann um eine Frage, die zusätzlich zum Problem des Kriteriums für die Unterscheidung zwischen Semantik und Pragmatik erörtert wird, wenn angenommen wird, dass die Semantik und die Pragmatik nicht als die Theorie des Gesagten und die Theorie des Gemeinten zu definieren sind.

6 Mit »Semantik« und »Pragmatik« sind manchmal Bedeutungs-Aspekte gemeint, manchmal aber auch Theorien oder Betätigungsfelder. Ich gehe davon aus, dass dies vorläufig nicht für Verwirrung sorgt, komme aber weiter unten (gegen Schluss von Abschnitt 3 des vorliegenden Kapitels) auf diesen Punkt zurück.

7 Szabó 2005, S. 1. Szabó bezieht sich im Zitat auf Charles Morris' Unterscheidung zwischen Semantik und Pragmatik, die weiter unten im vorliegenden Kapitel rekonstruiert wird.

8 Bach 2006, S. 463 . 
Und bei Ken Turner lesen wir:

The history of this distinction [between semantics and pragmatics] since Frege has not been particularly auspicious. On the one hand the legitimacy of the distinction has been denied by a vociferous philosophical community which takes its lead from remarks by the later Wittgenstein and which to this day proclaims that meaning is use [...]. On the other hand, those who have acknowledged the legitimacy of the distinction have drawn it in many non-compatible and nonequivalent ways. ${ }^{9}$

Avner Baz schreibt in seiner Verteidigung der Ordinary Language-Philosophie sogar selbst, dass die Vertreterinnen und Vertreter diese Philosophie-Konzeption nicht viel von der Unterscheidung zwischen Semantik und Pragmatik halten:

OLP radically questions these categories [semantics and pragmatics] and their philosophical usefulness. ${ }^{10}$

Und Recanati stellt die Sachlage so dar, dass die Ordinary-LanguagePhilosophinnen und -Philosophen als BegründerInnen der heutigen Pragmatik die Semantik nicht als gleichermaßen respektablen Forschungszweig anerkannten und dass der Umstand, dass der Streit um die Legitimität der Semantik und der Pragmatik mittlerweile beigelegt worden ist und heute allerseits eingeräumt wird, dass beide Disziplinen mit ihren unterschiedlichen Untersuchungszielen eine Daseinsberechtigung haben, unter anderem darauf zurückzuführen ist, dass die PragmatikerInnen von der Gebrauchstheorie der Bedeutung Abstand genommen haben:11

There are two main reasons for the new situation. On the one hand semanticists, in moving from artificial to natural languages, have given up Carnap's idea that the semantic relation between words and the world can be studied in abstraction from the context of use. [...] On the other hand those working in pragmatics no longer hold that 'meaning is use'. Instructed by Grice, they systematically draw a distinction between what a given expression means, and what its use means or conveys, in a particular context (or even in general). ${ }^{12}$

9 Turner 1997, Abschnitt 2. Hervorhebung von mir.

$10 \quad$ Baz 2012, S. 138.

11 Vgl. Recanati 2004b, S. 1-3.

12 Recanati 2004b, S. 2f. 


\section{Pragmatische Phänomene und die Gebrauchstheorie der Bedeutung}

Einige paradigmatische Beispiele für die Unterscheidung zwischen Semantik und Pragmatik hängen mit dem Unterschied zusammen, auf den Recanati im letzten Satz des obigen Zitats Bezug nimmt: dem Unterschied zwischen der Bedeutung eines Ausdrucks und dem, was der Gebrauch dieses Ausdrucks in einem bestimmten Kontext bedeutet. ${ }^{13}$ Betrachten wir etwa Grices berühmtes Evaluations-Beispiel:

I am reporting on a pupil at Collections. All I say is "Jones has beautiful handwriting and his English is grammatical." We might perhaps agree that there would here be a strong, even overwhelming, implication that Jones is no good at philosophy. $[\ldots][\mathrm{T}]$ he utterance of the sentence "Jones has beautiful handwriting etc." does not standardly involve the implication here attributed to it; it requires a special context (that it should be uttered at Collections) to attach the implication to its utterance. ${ }^{14}$

In einem bestimmten Kontext kann man jemandem mit der Äußerung des Satzes »Jones hat eine schöne Handschrift und sein Englisch ist grammatikalisch korrekt« zu verstehen geben, dass Jones philosophisch nicht besonders talentiert ist, obwohl dies offensichtlich nicht zur Bedeutung dieses Ausdrucks gehört. Ist es für Vertreterinnen und Vertreter der Gebrauchstheorie unmöglich, diesem Umstand Rechnung zu tragen? Diese Frage ist klar zu verneinen. Eine Vertreterin der Gebrauchstheorie setzt, grob gesagt, die konventionelle Bedeutung eines Ausdrucks mit der etablierten oder korrekten Art und Weise der Verwendung dieses Ausdrucks gleich. Was der Ausdruck »Jones hat eine schöne Handschrift $u s w$. « bedeutet, wird der Gebrauchstheorie zufolge dadurch festgelegt, wie dieser Satz oder seine Bestandteile etablierter- oder korrekterweise gebraucht werden. ${ }^{15}$ Wenn wir beschreiben, wie Ausdrücke wie »eine schöne Handschrift haben « oder »grammatikalisch korrektes Englisch sprechen « korrekterweise verwendet werden, werden wir natürlich an keiner Stelle die philosophische Eignung einer Person ansprechen, der diese Attribute zugeschrieben werden. Folglich gehört die oben genannte Aussage oder Andeutung betreffend die philosophische Eignung von Jones auch der

13 Recanatis eingeklammerte Ergänzung lasse ich vorläufig unberücksichtigt, komme aber weiter unten darauf zurück.

14 Grice 1961, S. 129f.

15 Auf die Frage, inwiefern man auch auf der Ebene von Sätzen (und nicht bloß auf der Ebene von Wörtern oder Redensarten) von einem etablierten Gebrauch bzw. von Regeln der Verwendung sprechen kann, gehe ich im Abschnitt 6 des vorliegenden Kapitels ein. 
Gebrauchstheorie zufolge nicht zur Bedeutung des Ausdrucks »Jones hat eine schöne Handschrift und sein Englisch ist grammatikalisch korrekt«. Darüber, wie eine solche Andeutung oder Implikatur, mit Grice gesprochen, zustande kommt, ist die Gebrauchstheorie der Bedeutung keine Theorie. Vertreterinnen und Vertreter der Gebrauchstheorie werden - ebenso wie die Verfechter anderer Bedeutungstheorien - gewisse allgemeine, unserer Kommunikation zugrunde liegende Prinzipien oder kommunikative Strategien anführen, um zu erklären, weshalb und unter welchen Umständen mit der oben besprochenen Äußerung eine Einschätzung der philosophischen Eignung von Jones kommuniziert werden kann. ${ }^{16}$ Die Annahme, auf der Grundlage der Gebrauchstheorie könne nicht zwischen der Bedeutung eines Ausdrucks und dem, was mit der Äußerung dieses Ausdrucks in einem einzelnen Kontext vermittelt werde, unterschieden werden, ist offensichtlich falsch.

Mit der eingeklammerten Ergänzung in der oben zitierten Passage aus »Pragmatics and Semantics « kommt ein weiterer Unterschied zur Sprache, dem Vertreterinnen und Vertreter der Gebrauchstheorie Recanati zufolge nicht Rechnung tragen können, nämlich der Unterschied zwischen dem, was ein Ausdruck bedeutet, und dem, was seine Äußerung im Allgemeinen bedeutet oder (zusätzlich) vermittelt. Ich vermute, dass Recanati hier solche Fälle vorschweben, wie sie von Grice unter dem Stichwort »generalisierte konversationelle Implikaturen « besprochen werden. Wenn beispielsweise über einen Mann gesagt wird, er sei heute Abend »mit einer Frau verabredet«, dann wird damit nahegelegt, dass es sich bei dieser Frau nicht um dessen Ehefrau, Mutter, Schwester oder enge platonische Freundin handelt. ${ }^{17}$ Meines Erachtens lässt sich dieses Phänomen nicht adäquat als Unterschied zwischen der Bedeutung eines Ausdrucks und dem, was die Äußerung des Ausdrucks im Allgemeinen zum Ausdrucks bringt oder nahelegt, beschreiben. Wenn die Äußerung von »X ist mit einer Frau verabredet« im Allgemeinen nahelegt, dass diese Frau keine Verwandte oder enge Vertraute von $\mathrm{X}$ ist, dann gehört dies zur Bedeutung dieses Ausdrucks. ${ }^{18} \mathrm{Zu}$ einem gewissen Teil lässt sich dieser

16 Dass in der betreffenden Situation aufgrund dieser allgemeinen Prinzipien und Strategien die erwähnte Implikatur auftritt, hängt aber natürlich trotzdem mit der konventionellen Bedeutung zusammen, die der geäußerte Ausdruck hat.

17 Vgl. Grice 1989, S. 37.

18 Dass dies zur Bedeutung des Ausdrucks gehört, heißt nicht zwingend, dass es zum $\mathrm{Ge}$ sagten gehört und auch nicht, dass die Äußerung etwas Unwahres zum Ausdruck bringen würde, wenn der betreffende Mann etwa mit seiner Schwester verabredet wäre. Auf das Verhältnis zwischen Bedeutung und Gesagtem und auf die Beziehung zwischen Bedeutung und Wahrheit komme ich weiter unten zurück. 
Aspekt der Bedeutung von $॥ \mathrm{X}$ ist mit einer Frau verabredet« sicherlich auf die Regeln zur Verwendung des unbestimmten Artikels zurückführen, und in dem Umfang, in dem diese Erklärung unzureichend ist, ${ }^{19}$ haben wir es mit dem Unterschied zwischen der wörtlichen und der idiomatischen Bedeutung des komplexen Ausdrucks zu tun.

Beides, sowohl die wörtliche als auch die idiomatische Verwendung eines Ausdrucks, ergibt sich aus der etablierten oder korrekten Art und Weise, den Ausdruck zu verwenden, bloß dass die wörtliche Bedeutung unter Rückgriff darauf analysierbar ist, wie die Teilausdrücke etablierter- oder korrekterweise verwendet werden, während die idiomatische Bedeutung davon abhängt, wie der komplexe Ausdruck (im obigen Fall: »mit einer Frau verabredet sein«) gebraucht wird. Ein ähnlicher Fall wie der vorangehend besprochene, in welchem sich deutlicher eine wörtliche von einer idiomatischen Bedeutung unterscheiden lässt, wäre: »Ich habe jemanden kennengelernt.«

Wie sich gezeigt hat, kann die Gebrauchstheorie der Bedeutung beiden Abgrenzungen problemlos Rechnung tragen, auf die Recanati im obigen Zitat Bezug nimmt und die zu ziehen Recanati zufolge den von ihm so genannten »Pragmatikern« nur aufgrund ihres Verwerfens der Gebrauchstheorie möglich geworden ist. Beim ersten Unterschied, demjenigen zwischen der Bedeutung eines Ausdrucks und dem, was mit einer einzelnen Äußerung des Ausdrucks kommuniziert werden kann, handelt es sich um einen typischen Unterschied zwischen einem semantischen und einem pragmatischen Phänomen - wobei die Gebrauchstheorie nur auf die Analyse der ersten Sorte von Phänomenen gerichtet ist -, beim zweiten Unterschied, der im vorangehenden Abschnitt des vorliegenden Kapitels besprochen worden ist, handelt es sich um einen Unterschied zwischen zwei semantischen Phänomenen, die beide gebrauchstheoretisch beschreibbar sind, und zwar wörtliche und idiomatische Bedeutung.

19 Wie der Umstand zu erklären ist, dass die Äußerung des Satzes »Ich habe heute Morgen ein Haus betreten « nahelegt, dass es sich dabei um ein fremdes Haus handelte, während »Ich habe einen Finger gebrochen « nahelegt, dass es ein eigener Finger war, ist mir nicht klar, und die Erklärung, die Grice dafür in Studies in the Way of Words (1989, S. 38) gibt, leuchtet mir nur bedingt ein. Er bezieht sich darin auf Hörer-Erwartungen hinsichtlich dessen, welche Aspekte in einer Äußerung zu spezifizieren sind, und welche nicht. Was spezifiziert werden muss und was nicht, hängt jedoch, so scheint es mir, umgekehrt davon ab, welche Aspekte andeutungsweise bereits durch die Verwendung des unbestimmten Artikels (zusammen mit den Regeln für dessen Verwendung) spezifiziert werden. Im Falle der o. g. Beispiele des betretenen Hauses und des gebrochenen Fingers haben wir es jedenfalls vermutlich nicht mit idiomatischen Bedeutungen zu tun. 
Ob mit der Darlegung, dass es auf der Grundlage der Gebrauchstheorie möglich ist, semantische von pragmatischen Phänomenen zu unterscheiden, auch bereits gezeigt worden ist, dass Bach und Turner mit ihren oben zitierten Einschätzungen falsch liegen, könnte deshalb als fraglich betrachtet werden, weil »Semantik« und »Pragmatik« auf unterschiedliche Weisen gebraucht werden können: Es können damit wissenschaftliche Disziplinen gemeint sein (auf deren Teilnehmer Recanati mit "semanticists « und "pragmaticists" Bezug nimmt), es können aber auch (wie bei Bach) Theorien damit bezeichnet werden. Außerdem kann man mit »Semantik « und »Pragmatik« Betrachtungs- oder Beschreibungsweisen sprachlicher Ausdrücke meinen oder die untersuchten oder beschriebenen Phänomene selbst: So ist zum Beispiel gelegentlich von der »Semantik eines sprachlichen Ausdrucks« die Rede, womit die Bedeutung dieses Ausdrucks gemeint ist. Entsprechend kann man auch mithilfe der Adjektive »semantisch « und "pragmatisch" eine Sache mit einer Disziplin, einer Theorie, einer Betrachtungs- oder Beschreibungsweise oder einem sprachlichen Phänomen verbinden. ${ }^{20}$ Dass auf der Grundlage der Gebrauchstheorie eine Unterscheidung zwischen semantischen und pragmatischen Phänomenen möglich ist, so könnte man argumentieren, heißt noch nicht, dass man auch zwischen der semantischen und der pragmatischen Untersuchung der Sprache unterscheiden kann: Beides läuft aus der Sicht der Gebrauchstheorie auf eine Untersuchung des Sprachgebrauchs hinaus. Dieser Behauptung ist Folgendes entgegenzusetzen:

Zunächst einmal betrifft die obige Überlegung zum Zusammenfallen der Semantik mit der Pragmatik als wissenschaftliche Disziplinen nicht die Gebrauchstheorie im Besonderen. Vertreterinnen und Vertreter anderer Bedeutungstheorien identifizieren zwar Bedeutung und Gebrauch nicht miteinander, räumen aber sehr häufig ein, dass Ausdrücke ihre Bedeutung durch ihren Gebrauch erlangen, dass Bedeutung über Gebrauch superveniert und dass wir, um die Frage zu beantworten, welche Bedeutung ein Ausdruck hat, in erster Linie untersuchen müssen, wie der Ausdruck verwendet wird. ${ }^{21}$ Auch diesen Philosophinnen und Philosophen zufolge entspricht also die Untersuchung sprachlicher Bedeutung-ebenso wie die Untersuchung pragmatischer Phänomene - im Wesentlichen einer Untersuchung unseres Sprachgebrauchs. Wichtiger als dieser Umstand ist allerdings, dass unser Sprachgebrauch viele verschiedene Facetten hat, die man auch einzeln in den Blick nehmen kann:

\footnotetext{
20 Vgl. dazu auch Levinson 1983, S. 3, dessen Disambiguierung mit meiner allerdings nicht deckungsgleich ist.

21 Vgl. Whiting 2008, S. 136, Recanati 2004b, S. 444.
} 
Die Art und Weise, wie einzelne sprachliche Ausdrücke verwendet werden, ist ausschlaggebend für die sprachliche Bedeutung dieser Ausdrücke und lässt sich gesondert von den allgemeinen, unserer Kommunikation zugrunde liegenden Prinzipien untersuchen, auf denen pragmatische Phänomene basieren. Wenn wir »Semantik« und »Pragmatik« als Bezeichnungen für wissenschaftliche Disziplinen verwenden, dann befassen sich zwar beide Disziplinen mit unserem Sprachgebrauch, aber mit unterschiedlichen Aspekten desselben.

\section{$4 \quad$ Semantik vs. Pragmatik}

\subsection{Abgrenzungsvarianten}

Grices oben besprochener Evaluations-Fall (»Jones hat eine schöne Handschrift usw. «) ist ein gutes Beispiel für eine Äußerung, deren Bedeutung semantische und pragmatische Aspekte verbindet. Bevor wir prüfen können, ob es auf der Basis der Gebrauchstheorie möglich ist, der Semantik/PragmatikUnterscheidung nicht nur in diesem einen, klaren Beispiel, sondern in ihrer gesamten Spannbreite Rechnung zu tragen, müssen wir uns der Frage zuwenden, worauf die Unterscheidung zwischen Semantik und Pragmatik überhaupt abzielt und, damit verbunden, ob es (von der Gebrauchstheorie der Bedeutung unabhängige) Gründe dafür gibt, die Grenze zwischen den beiden Bereichen an einer bestimmten Stelle zu ziehen.

Charles W. Morris, auf den die Unterteilung der Semiotik in Syntaktik, Semantik und Pragmatik zurückgeht, ${ }^{22}$ definiert die Syntaktik als die Untersuchung der Beziehungen, die zwischen Zeichen untereinander bestehen, ${ }^{23}$ und die Semantik und die Pragmatik wie folgt:

One may study the relations of signs to the objects to which the signs are applicable. This relation will be called the semantical dimension of semiosis, symbolized by the sign ' $D_{\text {sem }}$; the study of this dimension will be called semantics. Or the subject of study may be the relation of signs to interpreters. This relation will be called the pragmatical dimension of semiosis, symbolized by ' $D_{\mathrm{p}}$ ' and the study of this dimension will be named pragmatics. ${ }^{24}$

\footnotetext{
22 Vgl. Posner 1981, S. 55.

23 Vgl. Morris 1966 [1938], S. 6 .

24 Morris $1966[1938]$, S. 6.
} 
Die Syntaktik befasst sich, so Morris weiter, beispielsweise mit Implikationsverhältnissen wie demjenigen zwischen »Tisch« und »Möbelstück«, die Semantik mit Designations- und Denotationsverhältnissen wie denjenigen zwischen »Tisch« und einer Art oder Klasse von Objekten (Designation) oder zwischen $»$ Tisch « und den Bezugsobjekten dieses Ausdrucks, also allen Tischen (Denotation), und die Pragmatik mit der Relation des Äußerns. ${ }^{25}$

Die oben erwähnten Relationen bedingen sich gegenseitig. Zeichen verfügen im Normalfall nur dann über eine Denotation oder Designation, wenn sie verwendet werden, und eine Entität ohne Bedeutung und ohne Regeln des Gebrauchs würden wir nicht als »Zeichen« (d. h. als Symbol, mit Peirce gesprochen) bezeichnen. ${ }^{26}$ Diese wechselseitige Abhängigkeit hindert uns aber natürlich nicht daran, semantische Verhältnisse (im Morris'schen Sinn) unabhängig von syntaktischen und pragmatischen Verhältnissen zu beschreiben, und dasselbe gilt in die anderen Richtungen. Dieser Punkt sollte auch vor dem Hintergrund der Gebrauchstheorie der Bedeutung betont werden: Dass Ausdrücke nur deshalb Bezugsobjekte (oder wenigstens eine »Designation « im oben explizierten Sinn) haben, weil sie eine Verwendung haben, heißt nicht, dass wir nicht darüber sprechen können, welche Bezugsobjekte oder welche Designation ein Ausdruck hat, ohne (explizit) über seinen Gebrauch zu sprechen. Morris' oben rekonstruierte Unterscheidung zwischen Semantik und Pragmatik lässt sich also auch aus der Perspektive der Gebrauchstheorie der Bedeutung nachvollziehen.

Im Zuge der Debatte um die Semantik/Pragmatik-Unterscheidung, die sich seit der Einführung dieser Unterscheidung im Jahr 1938 entwickelt hat, ist eine Vielzahl verschiedener Kriterien zur Abgrenzung des Gegenstandsbereichs der Semantik von demjenigen der Pragmatik vorgeschlagen worden; darunter die folgenden: ${ }^{27}$

25 Vgl. Morris 1966 [1938], S. 5, 7. Was sich Morris unter einer Untersuchung der »Relation des Äußerns« genau vorstellt, geht aus seinen Ausführungen an der betreffenden Stelle nicht hervor.

26 Vgl. Morris 1966 [1938], S. 4.

27 Vgl. z. B. die Aufzählungen in Levinson 1983 (Kap. 1), Turner 1997 (Abschnitt 2), Jaszczolt 2002 (S. 225), Recanati 2004b (passim). 


\begin{tabular}{lll}
\hline & Semantik & Pragmatik \\
\hline 1 & Bedeutung & Gebrauch \\
2 & Ausdrucksbedeutung & Äußerungsbedeutung \\
3 & Gesagtes & Gemeintes \\
4 & Bezugsobjekte und & Aspekte der Bedeutung, die keinen \\
& Wahrheitsbedingungen & Einfluss auf den Bezug und die \\
& & Wahrheitsbedingungen sprachlicher \\
& & Ausdrücke haben \\
5 & konventionelle Bedeutung & nicht-konventionelle Aspekte der \\
& & Bedeutung \\
6 & wörtliche Bedeutung & nicht-wörtliche Aspekte der Bedeutung \\
7 & kontextunabhängige Bedeutung & kontextabhängige Aspekte der Bedeutung \\
8 & Aspekte der Bedeutung, die auf & Aspekte der Bedeutung, die auf \\
& sprachlichen Regeln beruhen & Konversationsmaximen oder auf \\
& & Tendenzen und Strategien des \\
& & Sprachgebrauchs beruhen \\
9 & Sprachliche Bedeutung, mit & Indexikalität (wenigstens große Teile \\
& Ausnahme der Phänomene, die & davona), Implikaturen, Präsuppositionen, \\
& von der Pragmatik behandelt & Sprechakte, Aspekte der Diskursstruktur \\
& werden & \\
\hline & &
\end{tabular}

a Manche PhilosophInnen und LinguistInnen vertreten die Auffassung, dass es Spezialfälle von indexikalischen Ausdrücken, wie z. B. »ich«, gibt, deren Bezug unabhängig von Sprecherabsichten u. Ä. festgelegt ist und die deshalb nicht in den Bereich der Pragmatik fallen.

Ich werde mich in der vorliegenden Abhandlung nicht mit der Frage auseinandersetzen, in welchem Maße sich die einzelnen Unterscheidungsansätze überschneiden, ${ }^{28}$ und gehe stattdessen auf einige Probleme ein, mit denen die verschiedenen Vorschläge konfrontiert sind.

28 Ein interessanter Punkt in diesem Zusammenhang ist vielleicht, dass die wörtliche Bedeutung sprachlicher Ausdrücke nicht mit ihrer konventionellen Bedeutung deckungsgleich ist. Der Unterschied zwischen diesen beiden Kategorien tritt nicht nur im Zusammenhang mit Redewendungen zutage (die wörtliche Bedeutung von »Wink mit dem Zaumpfahl« ergibt sich aus den Bedeutungen der im Ausdruck vorkommenden Wörter, während zur konventionellen Bedeutung auch die idiomatische Bedeutung gehört), sondern beispielsweise auch im Zusammenhang mit dem Phänomen der Ironie. Diese gilt allgemein als ein pragmatisches Phänomen, es gibt allerdings auch feste, 


\subsection{Interne Probleme}

Zunächst einmal kann eine Ausdrucksbedeutung natürlich mit der Bedeutung einer Äußerung des entsprechenden Ausdrucks übereinstimmen (vgl. Kriterium 2) (dies wäre z. B. dann der Fall, wenn ich jetzt den Satz »Der Mount Everest ist 8848 Meter hoch« äußere), und noch häufiger stimmt das Gesagte mit dem Gemeinten überein (vgl. Kriterium 3) (dies dürfte sogar der Normalfall sein) ${ }^{29}$ Angesichts dieses Umstands wäre es wohl adäquater, zu sagen, die Pragmatik befasse sich mit denjenigen Teilen der Äußerungsbedeutung oder des Gemeinten, die über die Ausdrucksbedeutung oder das Gesagte hinausgehen oder von ihnen abweichen. Aus einem analogen Grund habe ich in den Vorschlägen (4) bis (6) jeweils die Formulierung »Aspekte der Bedeutung « gewählt, die nicht unterstellt, es gebe neben der konventionellen, der wörtlichen oder der kontextunabhängigen Bedeutung eines Ausdrucks jeweils auch noch nicht-konventionelle, nicht-wörtliche oder kontextabhängige Bedeutungen, die von den ersteren gesondert untersucht werden könnten.

Ein weiteres wiederkehrendes Problem betrifft die Frage nach der Bestimmtheit der einzelnen in der Tabelle aufgeführten Kategorien. Dieses Problem tritt besonders drastisch im Falle des Kriteriums 1 auf: Als »Bedeutung « simpliciter werden je nach Kontext unterschiedliche Phänomene bezeichnet, unter anderem diejenigen, für die auch die Bezeichnungen »Ausdrucksbedeutung«, »konventionelle Bedeutung «, »wörtliche Bedeutung « und beispielsweise auch »Äußerungsbedeutung « etabliert sind, und diese verschiedenen Kategorien fallen klar auseinander. Auch mit »Gebrauch « kann verschiedenerlei gemeint sein;neben einzelnenVerwendungen eines Ausdrucks insbesondere die Art(en) und Weise(n), wie ein Ausdruck verwendet wird, und die Art und Weise, wie ein Ausdruck verwendet werden sollte resp. etablierter- oder korrekterweise verwendet wird. ${ }^{30}$ Das Kriterium 1 ist also hochgradig erläuterungsbedürftig. Wie problematisch es ohne Erläuterung ist, kann man auch daran sehen, dass viele Definitionen der Pragmatik deren Gegenstandsbereich bestimmen als »denjenigen Teil der Bedeutung, der ... «.31 »Bedeutung « für sich allein kann, wie bereits weiter oben erwähnt, unter anderem Äußerungsbedeutung heißen, und mit der Äußerungsbedeutung setzen sich beide Disziplinen auseinander,

ironische Wendungen, im Falle derer die Ironie eindeutig Teil der konventionellen Bedeutung ist, z. B. »Das ist mir eine schöne Bescherung!« (vgl. zu diesem Beispiel FehlauerLenz 2008, S. 106).

29 Vgl. Baker/Hacker 2005a, S. 359, Hacker 1996, S. 699.

$30 \quad$ Vgl. Whiting, S. 137 f.

31 Vgl. z. B. die so genannte »Gazdar-Formel«: »PRAGMATICS = MEANING - TRUTH CONDITIONS « (Gazdar 1979, S. 2, zitiert aus Levinson 1983, S. 12). Der Gedankenstrich ist als »minus« zu lesen. 
die Semantik und die Pragmatik. Die Implikatur in der oben beschriebenen Situation der Evaluation des Studenten Jones ist zum Beispiel meines Erachtens klar Bestandteil der Bedeutung der Äußerung des Professors. ${ }^{32}$

Die Bestimmung des Gegenstandsbereichs der Semantik als das Gesagte (Kriterium 3) stellt sich bei näherer Betrachtung ebenfalls als unscharf heraus. In der einschlägigen Literatur tritt häufig die Definition auf, das Gesagte entspreche der konventionellen Bedeutung des geäußerten Satzes, ergänzt durch solche Kontextinformationen, die notwendig sind, um etwas Propositionales (d. h. Wahrheitsfähiges) zu erhalten. ${ }^{33}$ Recanati bezeichnet diesen Prozess der Ergänzung zu etwas Propositionalem als Sättigung (saturation). Der Sättigung bedürfen zum Beispiel Äußerungen, die indexikalische Ausdrücke enthalten oder die sich elliptisch auf eine gestellte Frage beziehen (»Wann hast Du Geburtstag? - »Am zweiten Mai.« - »Er hat gesagt, dass er am zweiten Mai Geburtstag hat.«). ${ }^{34}$ Nicht ganz klar ist, ob auch bestimmte Implikaturen im Grice'schen Sinn Teil des Gesagten sein können. Bei Ehrhardt/Heringer lesen wir:

Wenn jemand (1) äußere, dann implikatiere er, dass nicht alle Offiziere unterschrieben haben.

(1) Einige Offiziere haben unterschrieben.

Es wäre nun aber - jedenfalls so weit ich Deutsch kann - absurd zu behaupten, das sei mit der Äußerung nicht gesagt worden. ${ }^{35}$

Mit diesem Urteil bin ich nicht einverstanden. Wenn jemand den Satz »Einige Offiziere haben unterschrieben « äußert, gibt er seinen Zuhörern zwar unter anderem zu verstehen, dass nicht alle Offiziere unterschrieben haben, aber ich würde bestreiten, dass dies Teil dessen ist, was er sagt. Anders sieht es unter Umständen im folgenden, von Recanati beschriebenen Fall aus:

From a minimalist point of view, the $[. .$.$] sentence [...] 'I've had breakfast', ex-$ presses the proposition that $S$ (the speaker) has had breakfast before $t^{*}$ (the time of utterance). Strictly speaking this proposition would be true if the speaker had had breakfast twenty years ago and never since. This is clearly not what the speaker means (when she answers the question 'Do you want something to eat?'

32 Recanati (2001, S. 75) meint ebenfalls, dass zur Bedeutung von Äußerungen auch Implikaturen gehören.

33 Vgl. z. B. Recanati 2001, S. 76. Turner 1997 (Abschnitt 3.4) verortet diese Position bei Carston (1988); ich kann im betreffenden Text allerdings keine einschlägige Stelle finden.

34 Vgl. Recanati 2004a, S. 7.

35 Ehrhardt/Heringer 2011, S. 83. 
and replies 'I've had breakfast'); she means something much more specific, namely that she's had breakfast on that very day (that is, the day which includes $\left.\mathrm{t}^{*}\right){ }^{36}$

Wenn ich meine Arbeitskollegin frage, ob sie etwas essen möchte, und sie entgegnet: »Ich hatte ein großes Frühstück«, dann würde ich es zwar seltsam finden, unmittelbar darauf einem anwesenden Kollegen, der die Antwort nicht verstanden hat, zu erklären: »Sie hat gesagt, sie habe heute ein großes Frühstück gehabt«, aber wenn ich später beim Mittagessen gefragt werde, wie meine Kollegin begründet habe, dass sie nicht mitkomme, hätte ich kein Problem damit, diese Antwort zu geben (oder den geäußerten Satz sogar noch weiter zu paraphrasieren). Im Allgemeinen scheint mir der Kontext der Angabe des Gesagten für die Adäquatheit einer entsprechenden Formulierung eine entscheidende Rolle zu spielen: Wenn einem Sprecher $S$ ein amüsanter Versprecher unterläuft und eine andere Person im allgemeinen Gelächter fragt: »Was hat er gesagt? «, dann wäre das Gesagte in direkter oder indirekter Rede im genauen Wortlaut wiederzugeben. Es wäre jedoch normalerweise inadäquat, zwei Tage später zu behaupten: $\gg S$ hat ja vorgestern gesagt, dass $p \ll$, wenn klar ist, dass $S$ den Ausdruck mit der Bedeutung $p$ versehentlich geäußert hat. Je nachdem, in welchem Kontext und, damit verbunden, zu welchem Zweck das Gesagte angegeben respektive nach dem Gesagten gefragt wird, scheinen also mehr oder weniger Modifikationen erlaubt oder angebracht zu sein. ${ }^{37}$

Ein weiteres Problem hinsichtlich des Kriteriums 3 besteht darin, dass nicht ganz klar ist, inwiefern die Kategorie des Gesagten beispielsweise auf die Äußerung von Frage- oder Befehlssätzen anwendbar ist. ${ }^{38}$

\subsection{Adäquatheitsbedingungen für die Unterscheidung}

Angesichts dessen, dass Morris 1938 »Semantik« und »Pragmatik« als Kunstbegriffe eingeführt hat, also keiner zuvor existierenden Verwendungsweise dieser beiden Ausdrücke verpflichtet war, ist es eigentlich überraschend, dass sich um die Frage, wo die Grenze zwischen den beiden Disziplinen oder Phänomen-Typen adäquaterweise zu ziehen ist, eine Debatte entwickelt hat.

$36 \quad$ Recanati 2004a, S. 8.

37 Im Lichte dieser Ausführungen würde ich auch ein damit zusammenhängendes Beispiel aus Wittgensteins Philosophischen Untersuchungen differenzierend beurteilen: »Wer von einem Tag auf den andern verspricht >Morgen will ich dich besuchen $<$ - sagt der jeden Tag das Gleiche; oder jeden Tag etwas anderes?« $(P U, \S 226)$. Je nach Kontext und Stoßrichtung der Frage nach der Identität des Gesagten kann sich diese Frage entweder auf den Wortlaut oder auf die ausgedrückte Proposition beziehen und wäre entsprechend in bestimmten Fällen zu bejahen, in anderen zu verneinen.

Vgl. Ehrhardt/Heringer 2011, S. 116. 
Im vorliegenden Abschnitt soll geklärt werden, auf welche Sorte von Argumenten sich die Teilnehmerinnen und Teilnehmer der Debatte beziehen, wenn sie begründen, weshalb die von ihnen befürwortete Unterscheidungsvariante das Label »Semantik/Pragmatik-Unterscheidung « mehr als die anderen Varianten verdient hat.

Szabó schreibt in seiner Einleitung zu Semantics versus Pragmatics:

The fact that we do not have a robust and widely agreed upon explicit conception of what that distinction really amounts to does not make the debates futile: perhaps the participants share a tacit and fairly rich underlying conception of the distinction, a conception that has yet to be adequately articulated. ${ }^{39}$

Die Idee, dass den verschiedenen an der Debatte Beteiligten im Grunde genommen ein und dieselbe Konzeption der Semantik/Pragmatik-Unterscheidung vorschwebt, halte ich für unplausibel. Dies unter anderem deshalb, weil sich in den Meinungsverschiedenheiten innerhalb der Diskussion um die Semantik/ Pragmatik-Unterscheidung deutlich manifestiert, dass die verschiedenen Beteiligten nicht darin übereinstimmen, welche Annahmen in Bezug auf die Charakteristika der beiden Bereiche sie aufzugeben bereit sind.

Auch wenn es nicht eine einzige Weise gibt, die Grenze zwischen Semantik und Pragmatik zu ziehen, denen allen an der Debatte beteiligten PhilosophInnen und LinguistInnen »eigentlich « zustimmen würden, nimmt ein Teil der Argumente, die in der einschlägigen Literatur vorgebracht werden, auf geteilte Überzeugungen oder Erwartungen hinsichtlich der beiden Bereiche Bezug. So wird beispielsweise gegen das oben genannte Kriterium 4 (die Semantik bestimme die Bezugsobjekte und Wahrheitsbedingungen sprachlicher Ausdrücke oder Äußerungen) eingewendet, es gebe Ausdrücke mit einer konventionellen Bedeutung, die weder einen Bezug noch Wahrheitsbedingungen haben (und auch keinen Beitrag zu den Wahrheitsbedingungen komplexer Ausdrücke leisten), zum Beispiel »Goodbye«, im Falle derer es inakzeptabel wäre, wenn die Semantik über sie nichts sagen könnte. ${ }^{40}$ Ein anderes Argument, das auf geteilte Annahmen der Diskussionsteilnehmer Bezug nimmt, zielt darauf ab, zu zeigen, dass bestimmte Unterscheidungsvarianten einzelne Phänomene, die als paradigmatisch für den Bereich der Pragmatik gelten (z. B. Präsuppositionen, Sprechakte und Implikaturen), dem Bereich der Semantik zuordnen. ${ }^{41}$

39 Szabó 2005, S. 7.

40 Vgl. Recanati 2004b, S. 445.

41 Vgl. Levinson 1983, S. 9 f. 
Ein weiterer typischer Kritikpunkt an bestimmten Vorschlägen zur Unterscheidung der Pragmatik von der Semantik, der bereits im Abschnitt 3.2 des vorliegenden Kapitels angeklungen ist, betrifft die mangelnde Eindeutigkeit der Abgrenzung des Gegenstandsbereichs der Pragmatik von demjenigen der Semantik (oder auch von demjenigen der Psycho- oder Soziolinguistik). ${ }^{42}$

Außerdem stellt beispielsweise Stephen Levinson an die Unterscheidung zwischen Semantik und Pragmatik die Ansprüche, (a) sie soll die Entdeckung neuer Arten pragmatischer Phänomene zulassen (dieses Kriterium wird etwa vom Kriterium 9 in der obigen Tabelle nicht erfüllt) ${ }^{43}$ und (b) der Bereich der Pragmatik soll nicht einfach negativ so definiert werden, dass in ihn alle Aspekte dessen fallen, was sprachliche Äußerungen vermitteln können, die nicht von einer bevorzugten semantischen Theorie erfasst werden. ${ }^{44}$ Die Pragmatik soll stattdessen bezugnehmend auf ein von allen pragmatischen Phänomenen geteiltes Merkmal bestimmt werden. ${ }^{45}$ Auf dieser Grundlage greift Levinson Abgrenzungsvorschläge wie die Nr. 4 in der obigen Tabelle an.

Recanati macht im Artikel »Pragmatics and Semantics« (2004) geltend, es sei sowohl die Auffassung etabliert, ein Bedeutungs-Phänomen sei in dem Maße semantisch, in dem es konventionell (im Gegensatz zu kontextabhängig) sei, als auch die Auffassung, ein Bedeutungs-Phänomen sei in dem Maße semantisch, in dem es einen Einfluss auf die Wahrheitsbedingungen (oder den Bezug) eines sprachlichen Ausdrucks oder einer Äußerung habe. ${ }^{46}$ Diese Auffassungen sind nicht nur gleichermaßen etabliert, sondern viele Personen stimmen ihnen beiden gleichzeitig zu. Allerdings sind die beiden Kriterien nicht koextensional. So haben zum Beispiel von Grice so genannte »konventionelle Implikaturen « einer verbreiteten Auffassung nach keinen Einfluss auf die Wahrheitsbedingungen von Äußerungen, ${ }^{47}$ und umgekehrt

42 Vgl. Levinson 1983, S. 10, 25.

43 Vgl. Levinson 1983 , Seite 27.

44 Vgl. Gazdar-Formel, Fußnote 31. Auf die Tendenz, die Pragmatik auf diese Weise negativ zu definieren, nimmt auch Yehoshua Bar-Hillel in »Out of the Pragmatic Wastebasket« (1971) Bezug.

45 Vgl. Levinson 1983, S. 18.

46 Vgl. Recanati 2004b, S. 461.

47 Vgl. Recanati 2004b, S. 458f. Grice führt als Beispiele für Sätze mit konventionellen Implikaturen unter anderem »She was poor but she was honest« (1961, S. 127) und »He is an Englishman; he is, therefore, brave« (1989, S. 25) an. Der erste Satz implikiert, dass es einen Kontrast zwischen Armut und Ehrlichkeit gebe, der zweite, dass die Tapferkeit des betreffenden Akteurs eine Konsequenz daraus ist, dass er Engländer ist. Da die betreffenden Implikaturen auf die Bedeutung der Ausdrücke »but« und »therefore« zurückzuführen sind, und nicht auf Grices Konversationsmaximen, bezeichnet er sie als konventionell (im Gegensatz zu konversationell). 
haben so genannte Implizituren (ein Beispiel dafür ist der oben beschriebene Fall »Ich hatte [heute] ein großes Frühstück«) einen Einfluss auf die Wahrheitsbedingungen von Äußerungen, ${ }^{48}$ ohne konventionell zu sein. ${ }^{49} \mathrm{Ob}$ wir diese Sorte von Phänomenen dem Bereich der Semantik oder demjenigen der Pragmatik zurechnen, müssen wir, so Recanati, willkürlich entscheiden. ${ }^{50}$ Einigen an der Debatte Beteiligten ist das Festhalten an einer wahrheitskonditionalen Semantik wichtiger, andere wehren sich hartnäckig dagegen, die Auffassung aufzugeben, semantische Analysen müssten von Äußerungskontexten unabhängig sein, ${ }^{51}$ aber Argumente gibt es ab einem bestimmten Punkt in der Diskussion keine mehr.

Aus der Perspektive der Gebrauchstheorie ließe sich die Unterscheidung zwischen Semantik und Pragmatik ungefähr wie folgt ausbuchstabieren: Zur Semantik gehört die konventionelle Ausdrucksbedeutung, zur Pragmatik gehören diejenigen Aspekte von Äußerungsbedeutung, die nicht Teil der entsprechenden konventionellen Ausdrucksbedeutungen sind. Diese Abgrenzungsvariante könnte im Lichte der Ausführungen im vorliegenden Abschnitt unter anderem deshalb kritisiert werden, weil sie den Bereich der Pragmatik negativ bestimmt und weil sie bestimmte Phänomene, die üblicherweise zur Pragmatik gerechnet werden, dem Bereich der Semantik zuordnet (viele Präsuppositionen ergeben sich beispielsweise konventionell auf der Ebene des Ausdrucks und treten nicht erst im Zuge von Äußerungen auf). Wie triftig diese Argumente sind, wird im Rahmen der vorliegenden Abhandlung nicht diskutiert (mir erscheinen die betreffenden Desiderate an eine Abgrenzung zwischen Semantik und Pragmatik nicht besonders relevant). Stattdessen soll darauf eingegangen werden, auf welcher Überlegung Grices berühmter Einwand gegen die Gebrauchstheorie beruht, gemäß welchem Vertreterinnen und Vertreter dieser Bedeutungstheorie dazu gezwungen sind, bestimmte pragmatische Phänomene als semantisch aufzufassen.

48 Zumindest behauptet dies Recanati; Grice würde der These, Implizituren hätten einen Einfluss auf die Wahrheitsbedingungen der betreffenden Äußerungen, nicht zustimmen.

49 Vgl. Recanati 2004b, S. 459-461.

50 Ganz ähnlich heißt es bei Turner (1997, Abschnitt 2):»[C]onclusions on these questions are sometimes more a matter of theoretical consistency, methodological strategy and, sometimes, individual temperament than of fact «.

$5^{1} \quad$ Vgl. Recanati 2004b, S. 445f. 
Grice bespricht seinen Einwand gegen die Gebrauchstheorie unter anderem anhand des Beispiels »versuchen«. Wenn ich über eine Person sage, sie versuche, die Tür zu öffnen, dann implikiere ich, so Grice, dass (a) sie beim Öffnen der Tür Schwierigkeiten hat oder (b) zu erwarten ist, dass sie dabei Schwierigkeiten hat, oder (c) irgendjemand glaubt, dass sie dabei Schwierigkeiten haben könnte. Nur unter der Bedingung, dass die Person Schwierigkeiten hat oder dass von mir oder jemand anderem erwartet wird, dass sie Schwierigkeiten hat, ist es angemessen, das Wort »versuchen « im betreffenden Kontext zu verwenden. ${ }^{52}$ Die Frage, mit der sich Grice befasst und die er negativ beantwortet, ist, ob die Insinuierung von Schwierigkeiten, die mit der Verwendung des Ausdrucks »versuchen« verbunden ist, zur Bedeutung dieses Ausdrucks gehört oder nicht. Grice zufolge handelt es sich beim betreffenden Aspekt des Gebrauchs von »versuchen « nicht um ein semantisches, sondern um ein pragmatisches Phänomen. ${ }^{53}$

Vertreterinnen und Vertreter der Gebrauchstheorie würden hinsichtlich der oben genannten Frage zu einem anderen Ergebnis kommen als Grice. Die oben formulierte Adäquatheitsbedingung für die Verwendung des Ausdrucks »versuchen« hat einen Einfluss auf die konventionelle Bedeutung dieses Wortes: Sie gehört zu den für die Bedeutung dieses Ausdrucks konstitutiven Regeln. Über eine Person, die nicht realisiert, dass Äußerungen von Sätzen der Form » $S$ (hat) versucht zu $\varphi$-en« nahelegen, dass $S$ das $\varphi$-en Schwierigkeiten bereitet hat, würden wir nicht sagen, dass sie die Bedeutung dieses Satzes versteht. In dieser Hinsicht unterscheidet sich das »versuchen«-Beispiel deutlich vom oben beschriebenen Handschriften-Beispiel: Wenn jemand hört, wie der Professor die Frage nach der philosophischen Eignung von Jones mit »Er hat eine schöne Handschrift« beantwortet, und nicht versteht, dass der Professor gerade ein negatives Urteil hinsichtlich von Jones' philosophischen Fähigkeiten kommuniziert hat, würden wir nicht sagen, dass diese Person den geäußerten Ausdruck nicht verstanden hat - obwohl wir evt. sagen würden, dass sie die Äußerung nicht (vollständig) verstanden hat. ${ }^{54}$ (Damit wir über die Person sagen würden, dass sie die (kontextunabhängige) Bedeutung der

\footnotetext{
52 Vgl. Grice 1989 , S. $6 f$.

53 Vgl. Grice 1989, S. 15 .

54 Auch Grice trägt dem Umstand Rechnung, dass wir es beim Versuchen-Beispiel mit einem anders gelagerten Fall zu tun haben als beim Handschriften-Beispiel. Ersteres ist für ihn ein Beispiel für eine generalisierte konversationelle Implikatur, Letzteres ein Beispiel für eine partikuläre konversationelle Implikatur (vgl. Grice 1989, S. 37).
} 
Antwort (und der gestellten Frage) verstanden hat, müsste sie im Übrigen erkennen, dass die Antwort (vordergründig) nicht zur Frage passt.)

Die Argumente, die Grice dafür anführt, den oben dargelegten Aspekt des Gebrauchs von »versuchen « nicht als semantisches, sondern als pragmatisches Phänomen zu betrachten, sind meines Erachtens nicht stichhaltig. Das wichtigste Argument von Grice zielt darauf ab, zu zeigen, dass das NichtErfülltsein der oben genannten Adäquatheitsbedingung für die Verwendung von »versuchen« (das Vorliegen oder die Erwartung von Schwierigkeiten) keinen Einfluss auf den Wahrheitswert von Äußerungen von Sätzen der Form $» S$ (hat) versucht zu $\varphi$-en « hat: $^{55}$ Wenn eine Person (absichtlich) eine Tür öffnet und ich hinterher über sie sage: »Sie hat versucht, die Tür zu öffnen«, so Grice, dann ist meine Äußerung wahr, auch wenn weder die Person selbst noch irgendein Zuschauer oder ich respektive meine Zuhörer die geringsten Zweifel daran hatten bzw. haben, dass es der Akteurin gelingen würde (bzw. gelungen ist), die Tür zu öffnen. Grice versucht dies unter Rückgriff auf diverse Beispiele zu belegen und richtet sich damit nicht nur gegen die Position, dass eine entsprechende Äußerung in der beschriebenen Situation den Wahrheitswert falsch hätte, sondern auch gegen die Position, dass eine entsprechende Äußerung weder wahr noch falsch wäre. Abgesehen davon, dass es Gründe dafür gibt, die Schlüssigkeit von Grices diesbezüglichen Argumenten zu bezweifeln, ${ }^{56}$ ist es für eine Vertreterin der Gebrauchstheorie überhaupt nicht nötig, zu zeigen, dass Äußerungen der beschriebenen Art entweder falsch oder weder wahr noch falsch sind. Dass der zur Diskussion stehende Aspekt Teil der Bedeutung von »verstehen« ist, kann nämlich auch stimmen, wenn die Erfülltheit oder Nicht-Erfülltheit der oben genannten Adäquatheitsbedingungen keinen Einfluss auf die Wahrheitsbedingungen von entsprechenden Äußerungen hat: Die Frage »Gehört dieser Aspekt zur Bedeutung von >versuchen<? « ist nicht gleichbedeutend mit der Frage »Hat dieser Aspekt einen Einfluss auf die Wahrheitsbedingungen von Sätzen der Form $>S$ (hat) versucht zu $\varphi$-en $<$ ?, und man kann die erste Frage bejahen und die zweite gleichzeitig verneinen. ${ }^{57}$

$\mathrm{Ab}$ und an klingt in Grices Argumentation zur Frage, ob es sich bei der Insinuierung von Schwierigkeiten bei der Verwendung des Wortes »versuchen«

55 Vgl. z. B. Grice 1989, S. 3, 10, 20 und im Zusammenhang mit einem anderen, aber analog gelagerten Fall S. 230.

56 Vgl. dazu z. B. Hanfling 200o, S. 18o, Glock 1996b, S. 218-220.

57 Dass es Aspekte sprachlicher Bedeutung gibt, die keinen Einfluss auf die Wahrheitsbedingungen entsprechender Äußerungen haben, kann meines Erachtens am einfachsten anhand von Pejorativen plausibel gemacht werden: »Hund « bedeutet nicht dasselbe wie »Köter«, auch wenn man »Hund « in allen möglichen Kontexten (in welchen das Wort verwendet und nicht erwähnt wird) salva veritate durch »Köter« ersetzen kann. 
um ein semantisches oder ein pragmatisches Phänomen handelt, zusätzlich die Idee an, wenn es möglich sei, die entsprechende Implikatur zusammen mit Implikaturen, die beim Gebrauch von anderen Ausdrücken auftreten, auf der Grundlage eines generellen, unserer ganzen Kommunikation zugrunde liegenden Prinzips zu erklären, sei dies gegenüber separaten Erklärungen für jeden einzelnen sprachlichen Ausdruck zu bevorzugen. ${ }^{58}$ Dafür, im zur Diskussion stehenden Fall eine schlankere Erklärung beziehungsweise eine Erklärung, die auf allgemeinere Gesetzmäßigkeiten Bezug nimmt, gegenüber einer ausführlicheren Erklärung zu bevorzugen, spricht aber meines Erachtens nichts, erst recht nicht, wenn wir unabhängige Gründe dafür haben, die betreffenden Phänomene zur Bedeutung der entsprechenden Ausdrücke zu zählen - nämlich etwa Gründe, die damit zusammenhängen, was man wissen muss, um die Bedeutung der entsprechenden Ausdrücke zu verstehen, bzw. damit, wie wir die Bedeutung der entsprechenden Ausdrücke erklären würden. Bei Grice findet sich kein Hinweis darauf, dass er einen unabhängigen Grund für die Wahl der schlankeren Erklärung hat. Die beiden im vorliegenden Abschnitt rekonstruierten Argumente von Grice für die These, der oben beschriebene Aspekt der Verwendung von »versuchen« sei nicht der Bedeutung dieses Wortes (resp. der Bedeutung von Sätzen, in denen es auftritt) zuzurechnen, sind also nicht überzeugend, und es gelingt Grice nicht, zu zeigen, dass die Gebrauchstheorie bestimmte pragmatische Phänomene zur Semantik zählt.

\section{Schluss}

Im vorliegenden Kapitel habe ich dafür argumentiert, dass es nicht stimmt, dass auf der Grundlage der Gebrauchstheorie sprachlicher Bedeutung keine Grenze zwischen Semantik und Pragmatik gezogen werden kann. Die Gebrauchstheorie identifiziert die Bedeutung sprachlicher Ausdrücke nicht mit allen Aspekten von deren Gebrauch, geschweige denn mit der Summe ihrer einzelnen Verwendungen in der Geschichte ihres Gebrauchs. Sondern die Bedeutung sprachlicher Ausdrücke wird mit der korrekten oder etablierten Weise ihres Gebrauchs gleichgesetzt. Dies lässt Raum dafür, dass man jemandem beispielsweise mit einer einzelnen Äußerung von »Jones hat eine schöne Handschrift und sein Englisch ist grammatikalisch korrekt« in einem geeigneten Kontext zu verstehen geben kann, dass Jones über kein besonderes Talent

58 Vgl. Grice 1989, S. 15, und im Zusammenhang mit einem anderen, aber analog gelagerten Fall S. 236, Grice 1961, S. 130-132. 
für Philosophie verfügt, obschon dies natürlich nicht Teil der Bedeutung des Ausdrucks »Jones hat eine schöne Handschrift und sein Englisch ist grammatikalisch korrekt « ist.

Es hat sich gezeigt, dass vor dem Hintergrund der Gebrauchstheorie nicht nur eine Unterscheidung zwischen semantischen und pragmatischen Aspekten der Bedeutung von Äußerungen möglich ist, sondern auch zwischen den Betätigungsfeldern der Semantik und der Pragmatik: Zwar sind Semantiker und Pragmatiker beide mit unserem Gebrauch sprachlicher Ausdrücke befasst, aber mit unterschiedlichen Aspekte desselben.

In Bezug auf die verschiedenen Kriterien, die in der entsprechenden Debatte zur Abgrenzung der Semantik von der Pragmatik ins Feld geführt wurden, hat sich herausgestellt, dass mehrere von ihnen überhaupt keine klare Grenze zwischen Semantik und Pragmatik bestimmen oder aus anderen Gründen problematisch sind. Die in der Debatte vorgebrachten Argumente für und gegen die einzelnen Kriterien nehmen teilweise auf geteilte Erwartungen hinsichtlich dessen Bezug, welche Phänomene eindeutig in den Bereich der Pragmatik oder eindeutig in den Bereich der Semantik fallen sollten. Alles in allem scheint es aber kein Knock-down-Argument dafür zu geben, eines der angeführten Kriterien gegenüber allen anderen vorzuziehen, und die Grenze, die sich auf der Grundlage der Gebrauchstheorie sprachlicher Bedeutung ziehen lässt, scheint keine relevanten Nachteile gegenüber den anderen Vorschlägen aufzuweisen.

Zum Schluss des Kapitels bin ich auf Grices Einwand gegen die Gebrauchstheorie der Bedeutung eingegangen, welchen ich anhand des Beispiels »versuchen « besprochen habe: Grice räumt ein, dass es nur dann angemessen ist, zu sagen, jemand habe versucht zu $\varphi$-en, wenn das $\varphi$-en dieser Person Mühe bereitet hat oder wenn zu erwarten gewesen ist, dass es der Person Mühe bereiten würde. Er besteht aber darauf, dass dieser Aspekt der Verwendung von »versuchen« keinen Einfluss auf die Bedeutung dieses Ausdrucks bzw. der Sätze, in denen er auftritt, hat. Ich habe dafür argumentiert, dass Grices Argumentation in diesem Zusammenhang nicht stichhaltig ist. Insbesondere muss ein Aspekt der Verwendung eines Ausdrucks contra Grice keinen Einfluss auf die Wahrheitsbedingungen von Sätzen haben, die den Ausdruck enthalten, damit er einen Einfluss auf die Bedeutung dieser Sätze haben kann.

Ich habe im vorliegenden Kapitel sowohl von der Bedeutung von Wörtern als auch von der Bedeutung von Sätzen gesprochen und beides mit entsprechenden Regeln des Gebrauchs in Verbindung gebracht. Damit sollte natürlich nicht über die Tatsache hinweggetäuscht werden, dass es wichtige Unterschiede zwischen der Bedeutung von Wörtern und derjenigen von Sätzen gibt, die den frühen Wittgenstein und Carnap sogar dazu veranlasst 
haben, den Ausdruck »Bedeutung« Wörtern vorzubehalten und Sätzen statt einer Bedeutung einen Sinn zuzuschreiben. Zum Beispiel können Sätzen, aber nicht Wörtern Wahrheitsbedingungen zugeschrieben werden, und Wörter, aber nicht Sätze haben manchmal eine Extension. ${ }^{59}$ Ein weiterer wichtiger Unterschied in den Bedeutungen von Wörtern und Sätzen besteht darin, dass unbestimmt viele Sätze gebildet werden können, die eine Bedeutung haben, ohne jemals verwendet worden zu sein. Demgegenüber haben »Wörter«, die noch nie gebraucht worden sind, für gewöhnlich keine Bedeutung (und sind mithin eigentlich auch keine Wörter). ${ }^{60}$

Nun könnte man sich natürlich fragen, ob man bei Sätzen, die noch nie verwendet worden sind, dennoch sinnvollerweise von Regeln ihres $\mathrm{Ge}$ brauchs sprechen kann. Mir erschließt sich nicht, weshalb dies nicht möglich sein sollte. Dass man einen Satz versteht, bedeutet, dass man weiß, unter welchen Bedingungen es korrekt oder angemessen wäre, ihn zu verwenden. ${ }^{61}$ Diese Bedingungen ergeben sich im Falle von Sätzen üblicherweise nicht aus ihrem etablierten Gebrauch, sondern aus dem etablierten Gebrauch ihrer Konstituenten und anderer, strukturgleicher Sätze. Aber natürlich gibt es auch bei Sätzen solche Bedingungen. Gerade wenn wir die Bedeutung von Sätzen bestimmen wollen, denen in der Philosophie ein besonderer Status zugeschrieben wird oder wurde, - zum Beispiel von analytischen Sätzen oder den Sätzen »Ich bin jetzt hier «62 oder »Es verhält sich soundso « ${ }^{63}-$, gibt es dafür wohl keine bessere Strategie, als die Frage zu beantworten, unter welchen Umständen man diese Sätze äußern würde. Und indem wir solche Umstände beschreiben, geben wir Regeln zum Gebrauch dieser Ausdrücke an. ${ }^{64}$

59 Ich sehe hier von Fregeanischen Auffassungen und Mögliche-Welten-Semantiken, nach welchen auch Sätze Bezugsobjekte haben, ab.

6o Man könnte Ausnahmefälle konstruieren, in welchen einem Wort stipulativ eine Bedeutung verliehen wird, das dann aber nie gebraucht wird.

$61 \quad$ Vgl. dazu auch Moore 1964 [1942], S. 16, und Warnock 1951, S. 318.

62 Dieser Satz soll synthetisch a priori sein. Vgl. z. B. Kaplan 1989, S. 508 f.

63 Dies ist laut Wittgenstein in TLP 4.5 die allgemeine Satzform; in $P U$ § 134 kritisiert er diese frühere Behauptung.

64 Vgl. $P U \S 117$. 